\title{
Covid-19 Complicated by Hemorrhagic Necrotizing Pancreatitis: A Case Report
}

\author{
Raymond Hou, $M D^{1}$, Shruti Koti, $B A^{2}$ and Danielle K DePeralta, $M D^{3}$ \\ ${ }^{1}$ Department of Emergency Medicine \& Critical Care, Long Island Jewish Medical Center, Northshore University, USA \\ ${ }^{2}$ Donald and Barbara Zucker School of Medicine, USA \\ ${ }^{3}$ Assistant Professor, Department of Surgery, Long Island Jewish Medical Center, Northshore University, USA
}

\begin{abstract}
Background: COVID-19 is an infectious disease that has been shown to cause an aggressive, viral pneumonia. In addition to worsening pulmonary function, the COVID-19 illness course may also be complicated by hypercoagulability and varying degrees of organ dysfunction. Here we describe a case of COVID-19 complicated by multi-organ dysfunction and acute necrotizing pancreatitis.

Case Presentation: A 57-year-old male with no past medical history presented with a week of flu-like symptoms, nausea, vomiting, and acute respiratory distress. Soon after presentation, the patient was intubated for profound hypoxia and went into cardiopulmonary arrest. Following resuscitation efforts, the patient sustained return of spontaneous circulation and was admitted to the medical ICU for further management. He quickly developed a range of metabolic and hematologic abnormalities, raising concern for dehydration, systemic inflammatory response, and multi-organ dysfunction. He was found to be COVID-19 positive by PCR testing. After 2 days in the MICU, the patient was successfully extubated; however, he demonstrated altered mental status and abdominal tenderness. CT abdomen/ pelvis demonstrated findings concerning for acute necrotizing hemorrhagic pancreatitis. There were no drainable fluid collections, and no role for operative intervention. The patient continued to worsen despite aggressive supportive care and 3 sessions of hemodialysis. Palliative care was eventually consulted, and after multiple goals of care discussions with the family, the patient was transferred to the medical floor for palliative and comfort measures where he ultimately expired one week later.
\end{abstract}

Conclusion: COVID-19 is an aggressive pulmonary infection that often leads to multi-organ dysfunction. Although care has primarily focused on the pulmonary system, including viral pneumonia, ARDS, and superimposed infections, it is important to recognize the various complications that may arise from patients who are critically ill with this infection. Necrotizing pancreatitis and other manifestations of organ dysfunction should remain on the differential for patients in the ICU.

\section{Keywords}

COVID-19, Coronavirus, SARS-CoV-2, Multiorgan dysfunction, Multiple organ failure, Pancreatitis, Necrotizing pancreatitis

\section{Abbreviations}

COVID-19: Coronavirus Infectious Disease, or SARS-CoV2; PCR: Polymerase Chain Reaction; MICU: Medical Intensive Care Unit; CT: Computed Tomography; ARDS: Acute Respiratory Distress Syndrome; ED: Emergency Department; DIC: Disseminated Intravascular Coagulation

\section{Introduction}

COVID-19 is an infectious disease caused by severe acute respiratory syndrome coronavirus 2 (SARS-CoV-2). The disease was first identified in December 2019 in Wuhan [1], and has since spread globally, resulting in a pandemic affecting nearly every country in the world. As of July 15,2020 , more than 13.3 million cases have been reported in more than 188 countries and territories, resulting in more than 580,000 deaths [2].
*Corresponding author: Raymond Hou, MD, Department of Emergency Medicine \& Critical Care, Long Island Jewish Medical Center, Northshore University, Queens, NewYork, USA

Accepted: August 01, 2020

Published online: August 03, 2020

Citation: Hou R, Koti S, DePeralta DK (2020) Covid-19 Complicated by Hemorrhagic Necrotizing Pancreatitis: A Case Report. J Gastrointest Hepat Surg 3(1):32-36 
The most commonly observed presentations are pulmonary in nature, ranging from asymptomatic infiltrates seen on chest radiograph, aggressive viral pneumonia, to ARDS [3]. To date, the exact pathophysiology is poorly understood, thought to involve a combination of entry through ACE2 lung receptors, cytokine storm with occasional hemophagocytic lymphohistiocytosis, and microthrombi that may lead to sudden cardiac or hypoxic events [4]. Recent data from the United States reports $14.2 \%$ of hospitalized patients required intensive care, of which many patients developed multi-organ dysfunction [5].

Here we describe a case of COVID-19 resulting in multi-organ failure and acute necrotizing pancreatitis. This case highlights the importance of considering a multitude of presentations throughout the clinical course of this infectious disease.

\section{Case Presentation}

A 57-year-old male with no past medical history presented to the ED with shortness of breath and respiratory distress. Of note, the patient had been seen in the ED one week prior for fever, chills, and shortness of breath. Since then, he had also developed nausea and vomiting. Soon after arrival on this presentation, the patient was intubated for profound hypoxia ( $\mathrm{SpO}_{2} 82 \%$ on non-rebreather mask), following which the patient was found to be in asystole. After cardiopulmonary resuscitation efforts, the patient sustained return of spontaneous circulation. The patient was started on a norepinephrine drip, phenylephrine drip, and sodium bicarbonate drip, and admitted to the medical ICU (MICU). A heparin drip and full-dose aspirin were also started at this time for presumed acute coronary syndrome.

Upon admission to the MICU, the patient had a temperature of $96.8^{\circ} \mathrm{F}$, heart rate of $82 \mathrm{bpm}$, blood pressure 99/79 $\mathrm{mmHg}$, mean arterial pressure of $83 \mathrm{mmHg}$, respiratory rate of $20 \mathrm{bpm}$, and oxygen saturation at $100 \%$. His physical exam was notable only for bilateral lung crackles. His abdomen was soft, non-distended, with normal bowel sounds and no organomegaly. Initial ventilator settings were PEEP of $15 \mathrm{~cm}$ $\mathrm{H}_{2} \mathrm{O}$, tidal volume $450 \mathrm{~mL}, \mathrm{FiO}_{2} 100 \%$, and these were adjusted as needed. Chest radiograph demonstrated clear lungs and endotracheal tube in good position. (Figure 1).

The patient had significant laboratory changes within a short time frame, from his initial encounter in the ED to his first morning in the MICU. His white count increased from $23.23 \times 10^{9} / \mathrm{L}$ to $40.83 \times 10^{9} / \mathrm{L}$, his platelets decreased from $310 \times 10^{9} / \mathrm{L}$ to $173 \times 10^{9} / \mathrm{L}$, and hemoglobin decreased from $20.4 \mathrm{~g} / \mathrm{dL}$ to $13.8 \mathrm{~g} / \mathrm{dL}$. Other notable labs upon admission to the MICU included sodium $180 \mathrm{mEq} / \mathrm{L}$, potassium $5.5 \mathrm{mEq} / \mathrm{L}$, BUN $154 \mathrm{mg} / \mathrm{dL}$, creatinine $9.17 \mathrm{mg} / \mathrm{dL}$, AST $1010 \mathrm{U} / \mathrm{L}$ and ALT $393 \mathrm{U} / \mathrm{L}$ (previously $93 \mathrm{U} / \mathrm{L}$ and $88 \mathrm{U} / \mathrm{L}$ respectively). Procalcitonin was $41 \mathrm{ng} / \mathrm{mL}$, which was presumed to be from a superimposed bacterial infection. Despite a normal chest radiograph on initial presentation, the patient was found to be COVID-19 positive by PCR testing.

At this point, the patient's clinical status and laboratory findings were presumed to be due to dehydration, shock liver, and reactive leukocytosis from resuscitative efforts. Hep- arin drip was continued at this time, due to early reports of microthrombi on autopsies resulting from hypercoagulable states. Institutional policy suggested heparin drips on patients with five times the upper limit of normal for $d$-dimers. The patient was started on pipercillin/tazobactam given con-

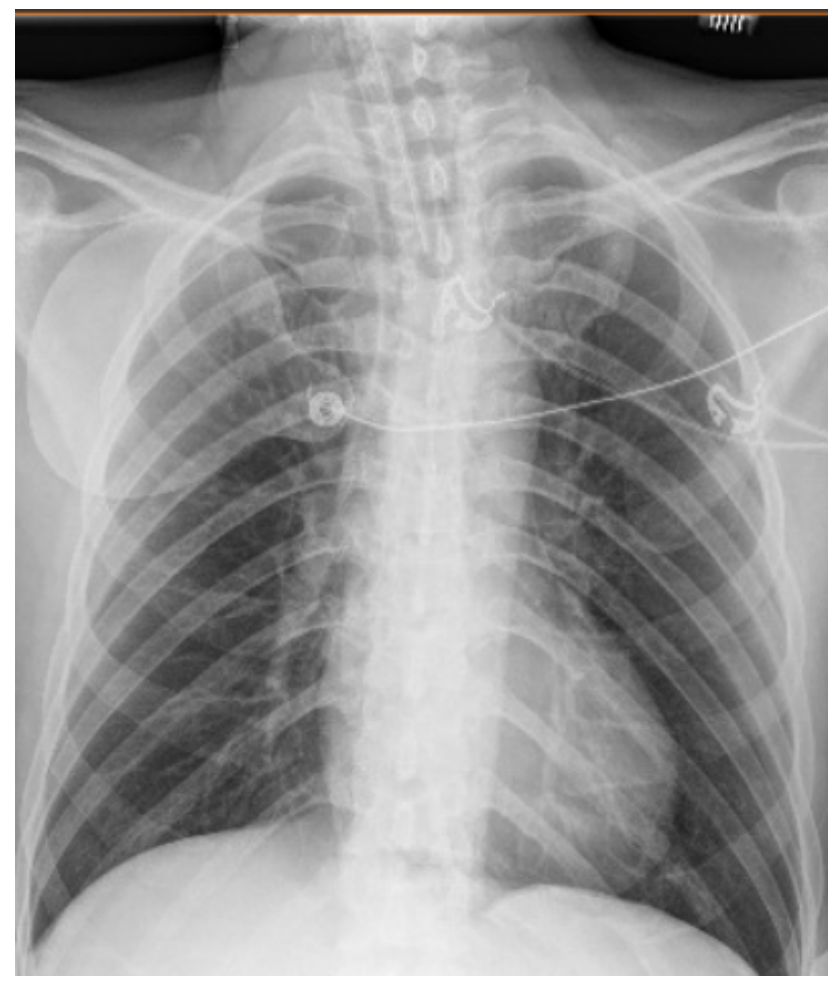

Figure 1: Chest Radiograph, day 1 of admission. A transcutaneous pacing pad projects over the right hemithorax. There are no focal consolidations, pleural effusions or pneumothorax. Endotracheal tube in place, with tip over the carina.

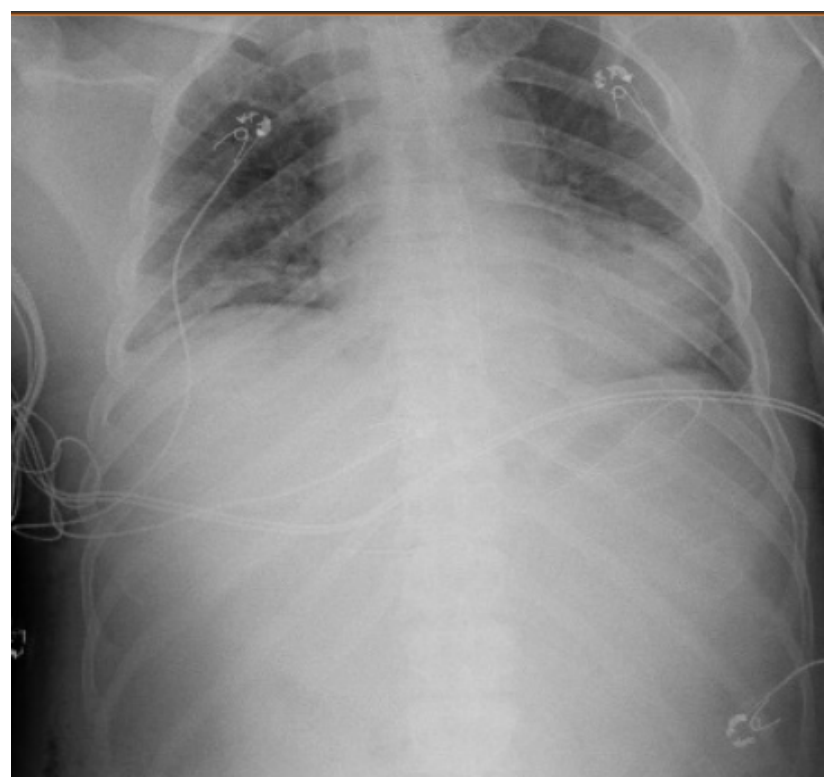

Figure 2: Chest Radiograph, day 4 of admission. Decreased lung volumes, with new mild perihilar opacities, possibly pulmonary vascular congestion or worsening atypical/viral infection consistent with COVID. 
Citation: Hou R, Koti S, DePeralta DK (2020) Covid-19 Complicated by Hemorrhagic Necrotizing Pancreatitis: A Case Report. J Gastrointest Hepat Surg 3(1):32-36

cern for superimposed bacterial infection, following which his lactate decreased from $17 \mathrm{mmol} / \mathrm{L}$ to $3.6 \mathrm{mmol} / \mathrm{L}$. Various treatments including hydroxychloroquine, azithromycin, IL-1/ IL-6 inhibitors, intravenous immunoglobulin, convalescent serum, vitamin $C$, and thiaminewere considered but these therapies were withheld due to concerns for dysrhythmias and multi-organ failure. Treatment with methylprednisolone was started for a total of 5 days.

After two days in the MICU, the patient was successfully extubated to $6 \mathrm{~L}$ nasal cannula. The patient was found to have poor mental status after extubation, though he was able to respond to simple commands. Given his acute renal injury and hypernatremia, nephrology services were consulted and the patient was started on a temporizing bumetanide drip with plans to initiate hemodialysis. There was also concern for DIC in the setting of multi-organ failure, therefore the heparin drip was discontinued, and vitamin $\mathrm{K}$ was administered. Follow up chest radiograph demonstrated low lung volumes with new mild perihilar opacities, possible pulmonary vascular congestion and/or worsening atypical/viral infection consistent with COVID (Figure 2).

On day 5 of patient's admission, the patient's hemoglobin acutely dropped from $9.6 \mathrm{~g} / \mathrm{dL}$ to $7.4 \mathrm{~g} / \mathrm{dL}$, and 2 units of packed red blood cells were administered. A physical examination revealed that he had new moderate abdominal tenderness without rebound or guarding. At this time, WBC were $32 \times 10^{9} / \mathrm{L}$. Chest CT was notable for extensive bilateral ground glass opacities consistent with known COVID-19 pneumonia (Figure 3). CT of the abdomen/pelvis without contrast revealed a diffusely edematous pancreas containing multiple areas of hypoattenuation with extensive peripancreatic inflammatory changes and fluid extending to the retroperitoneum. There was also hyperdense fluid, most consistent with blood, surrounding the tail of the pancreas and extending along the left paracolic gutter (Figure 4 and Figure 5). No gallstones were seen on right upper quadrant ultrasound. A lipase was not ordered, as the patient met 2 out of 3 criteria for pancreatitis (clinical exam and computed tomography findings). In addition, lipase and amylase levels would not

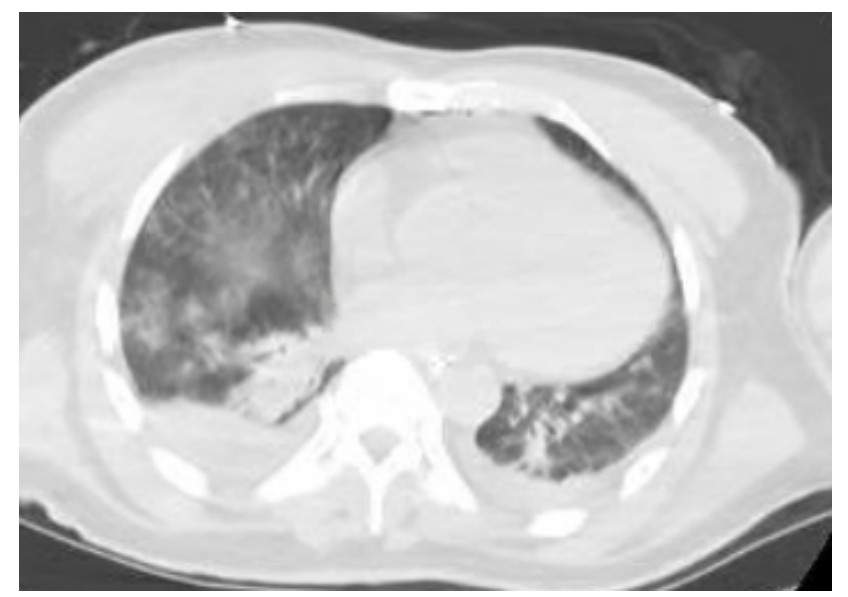

Figure 3: CT Chest (lung window), axial view, day 5 of admission. Pattern of diffuse scattered ground glass opacity compatible with reported atypical pneumonia/viral infection COVID-19. have changed management. Doppler exam demonstrated a normal caliber main portal vein without echogenic thrombus, and normal hepatopedal flow. General surgery and gastroenterology services were consulted and ongoing supportive care was recommended. There were no drainable fluid collections and no role for operative intervention at this point in the patient's course.

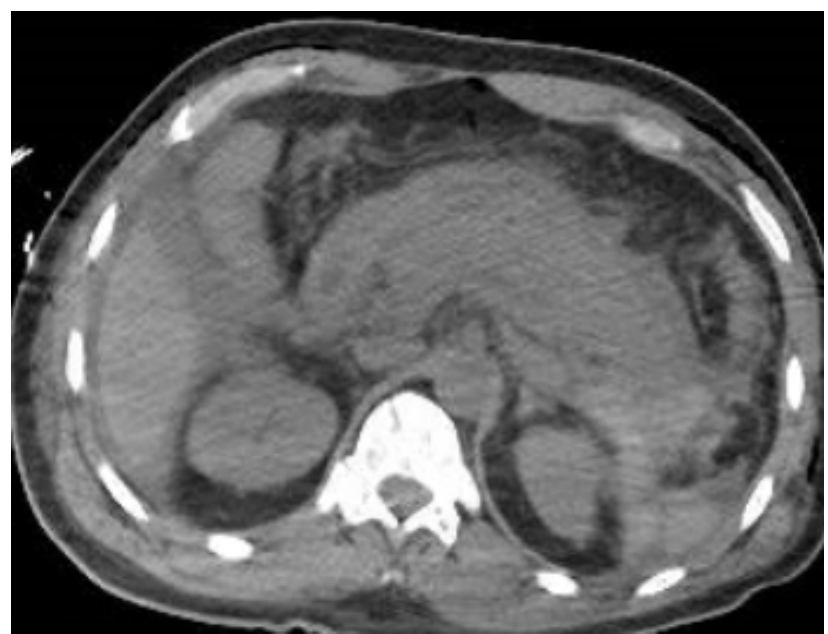

Figure 4: CT abdomen and pelvis, axial view, day 5 of admission. Findings concerning for extensive necrotizing hemorrhagic pancreatitis. At least two areas of hypoattenuation measuring 2.5 $\times 2.2 \mathrm{~cm}$ and $2.2 \times 1.7 \mathrm{~cm}$ in the body/tail the pancreas. These may represent walled off necrosis or developing abscesses. Mild diffuse colonic wall thickening, difficult to evaluate secondary to inadequate distention.

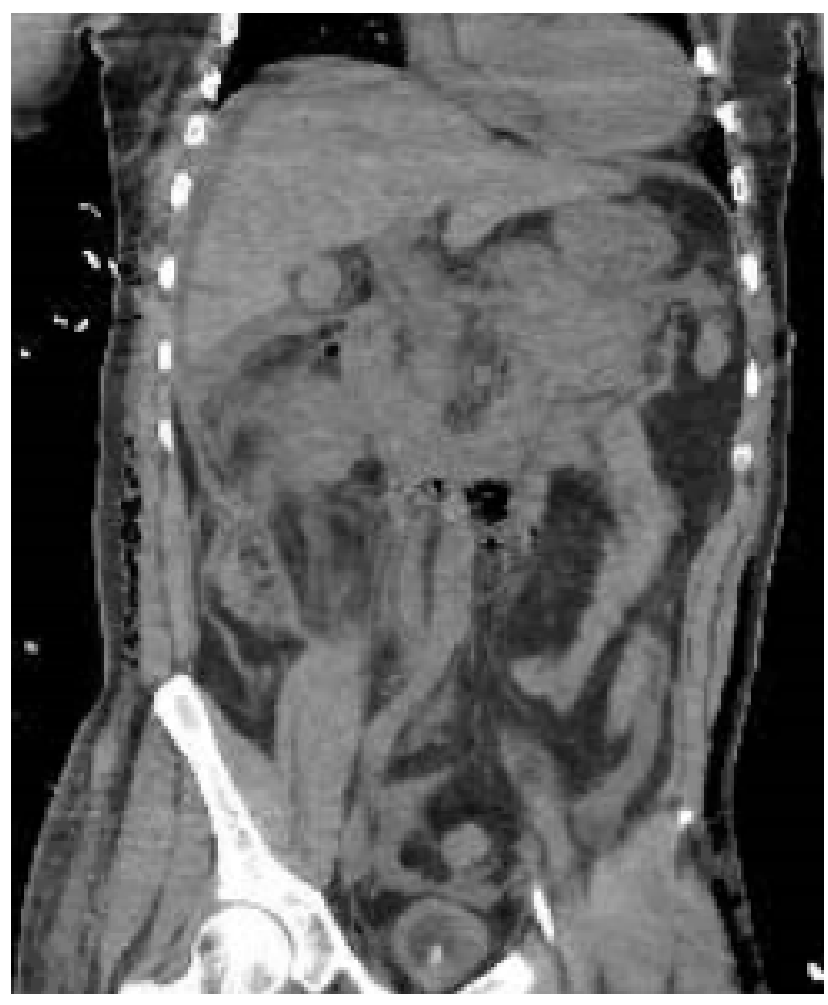

Figure 5: CT abdomen and pelvis, coronal view, day 5 of admission. See Figure 4 for report. 
Despite aggressive supportive care, the patient continued to have persistently worsening thrombocytopenia, anemia, hypotension, and renal failure. Potassium remained elevated at $7.3 \mathrm{mmol} / \mathrm{L}$ despite 3 sessions of hemodialysis. In addition, the patient's mental status worsened and his clinical status continued to decline. Palliative care service was eventually consulted to assist with goals of care conversations. Multiple goals of care discussions were undertaken among the palliative care service, the ICU team and the patient's family. Comfort care measures were implemented, and the patient was subsequently transferred to the medical floor, where he was started on a dilaudid drip and ultimately expired.

\section{Discussion}

COVID-19, although initially considered a respiratory illness, has now revealed itself as a disease that frequently leads to multi-organ dysfunction. This report illustrates a patient requiring critical care very soon after presentation, with an ultimate finding of hemorrhagic, necrotizing pancreatitis.

Although this patient was weaned off ventilatory support within 2 days, he had numerous other issues including renal failure, shock liver, disseminated intravascular coagulation (DIC), and severe hypernatremia. Known causes of DIC include sepsis, pancreatitis, liver disease, and acute respiratory distress syndrome (ARDS) [6]. This patient's hypotension and DIC were likely related to all four mechanisms, although his pancreatitis was only discovered on day 5 of admission when he began to exhibit signs of abdominal pain. It is unclear at what point this developed, as the patient was intubated and sedated for the first two days in the MICU. The hemorrhagic component of his pancreatitis was likely secondary to anticoagulation. More common etiologies of pancreatitis were excluded. A right upper quadrant ultrasound was negative for gallstones. The patient had no known history of alcohol abuse, family history of pancreatitis, and his serum triglycerides were $178 \mathrm{mg} / \mathrm{dL}$.

Mechanical ventilation has been associated with numerous organ system disorders that impact the outcome of critically ill patients, in part due to ventilation-induced injury and subsequent release of proinflammatory cytokines. Along with potential organ ischemia and catecholamine release, a systemic inflammatory response may induce pancreatitis via these mechanisms. Other potential mechanisms include ischemic injury due to vasoconstriction and splanchnic hypoperfusion causing redistribution of blood away from the splanchnic vascular bed. In this patient, whose ventilator settings included a relatively high PEEP and $100 \% \mathrm{FiO}_{2}$, it is possible that these contributed in part to his subsequent pancreatitis.

The early pathogenic mechanisms of acute pancreatitis involve premature activation of enzymes inside the pancreas, related to an impaired cytosolic $\mathrm{Ca}^{2+}$ homeostasis, as well as the release of pancreatic enzymes into the bloodstream. The vasodilatory effects of pro-inflammatory cytokines play a role in amplifying the inflammatory response [7]. Viral illnesses have been described to cause pancreatitis in less than $1 \%$ of cases [8] and rarely, influenza has been reported to be associated with pancreatitis [9]. Studies in mice have shown that certain influenza A subtypes infect and trigger apoptosis of pancreatic cells, as well as causing release of pro-inflammatory cytokines and chemokines [10]. However, there have as yet been no reports of pancreatitis associated with coronavirus infection.

Although human case reports of coronavirus and pancreatitis are lacking, one case report confirmed coronavirus disease and pancreatitis in ferrets. This particular case documents the occurrence of suppurative pancreatitis and necrotizing mesenteric steatitis in a young ferret concurrently, due to ferret coronavirus [11]. Though there are challenges in using animal models, early studies have shown similar viral replication and transmission in ferrets that may point to their potential use as an animal model for COVID-19 [12].

\section{Conclusion}

COVID-19 is an aggressive pulmonary infection that often leads to multi-organ dysfunction. Although much attention is given to treating superimposed infection, ventilatory and pulmonary support, it is important to monitor patients closely for additional, less common sequelae of the disease. Vigilance is especially key for patients being treated in an intensive care setting, where a reliable history and physical exam often cannot be fully performed due to patient sedation.

\section{Declarations}

\section{Consent for publication}

Patient deceased. IRB-approval waiver granted by institution.

\section{Availability of data and materials}

Not applicable.

\section{Competing interests}

The authors declare that they have no competing interests.

\section{Authors' contributions}

$\mathrm{RH}$ was involved in the clinical care of this patient. $\mathrm{RH}$ and SK were major contributors in writing the manuscript. All authors read, contributed to, and approved the final manuscript.

\section{Acknowledgments}

Not applicable.

\section{Research quality and ethics statement}

The authors of this manuscript declare that this scientific work complies with reporting quality, formatting and reproducibility guidelines set forth by the EQUATOR Network. The authors also attest that this clinical investigation was determined to not require Institutional Review Board/Ethics Committee review, and the corresponding protocol/approval number is not applicable.

\section{References}

1. Zhu N, Zhang D, Wang W, et al. (2020) A novel coronavirus from patients with pneumonia in China, 2019. N Engl J Med 382: 727-733. 
2. https://www.cdc.gov/coronavirus/2019-ncov/cases-updates/ index.html

3. Guan WJ, Ni ZY, Hu Y, et al. (2020) Clinical characteristics of coronavirus disease 2019 in China. N Engl J Med 382: 1708-1720.

4. Hoffmann M, Kleine-Weber H, Schroeder S, et al. (2020) SARSCoV-2 cell entry depends on ACE2 and TMPRSS2 and is blocked by a clinically proven protease inhibitor. Cell 181: 271-280.

5. Richardson S, Hirsch JS, Narasimhan M, et al. (2020) Presenting characteristics, comorbidities, and outcomes among 5700 patients hospitalized with COVID-19 in the New York City area. JAMA 26: 2052-2059.

6. Levi M, Ten Cate H (1999) Disseminated intravascular coagulation. N Engl J Med 341: 586-592.

7. Bhatia M, Wong FL, Cao Y, et al. (2005) Pathophysiology of acute pancreatitis. Pancreatology 5: 132-144.
8. Parenti DM, Steinberg W, Kang P, et al. (1996) Infectious causes of acute pancreatitis. Pancreas 13: 356-371.

9. Habib A, Jain A, Singh B, et al. (2016) H1N1 influenza presenting as severe acute pancreatitis and multiorgan dysfunction. Am J Emerg Med 34: 1911

10. Huo C, Xiao K, Zhang S, et al. (2018) H5N1 influenza a virus replicates productively in pancreatic cells and induces apoptosis and pro-inflammatory cytokine response. Front Cell Infect Microbiol 8: 386.

11. Wills SE, Beaufrère HH, Brisson BA, et al. (2018) Pancreatitis and systemic coronavirus infection in a ferret (Mustela putorius furo). Comparative medicine 68: 208-211.

12. Kim YI, Kim SG, Kim SM, et al. (2020) Infection and rapid transmission of SARS-COV-2 in ferrets. Cell host \& microbe 27: 704709. 